\title{
CHANGE DETECTION PERFORMANCE UNDER DIVIDED ATTENTION WITH DYNAMIC DRIVING SCENARIOS
}

\author{
Yi-Ching Lee, John D. Lee, Linda Ng Boyle \\ Department of Mechanical and Industrial Engineering \\ Center for Computer-Aided Design \\ University of Iowa \\ Iowa City, Iowa, USA \\ E-mail: yi-ching-lee@uiowa.edu
}

\begin{abstract}
Summary: This study investigated the effect of cognitive load on the relationship between confidence in detecting changes and actual change detection performance. Two experiments simulated glancing away from the roadway by periodically blanking the driver's view for one second. Experiments were conducted in a driving simulator where participants were asked to detect changes in the location and appearance of other vehicles while driving on a multi-lane suburban roadway. In addition, cognitive load was imposed using messages that participants were asked to listen to and answer questions about. Participants' sensitivity (d') to vehicle changes was calculated and compared with subjective ratings of confidence in detecting those changes. Results indicated a positive relationship between d' and confidence, suggesting that participants were aware of the factors that influenced their change-detection performance. However, the strength of the relationship was situation-dependent. The strength of the relationship decreased when the detection task was more difficult and in the presence of cognitive load.
\end{abstract}

\section{INTRODUCTION}

The extensive growth of the in-vehicle wireless communications industry has been accompanied by growing concern for the potential hazards of drivers using wireless communication devices while driving. For example, for drivers aged 16 to 24, there is an increase from 5\% in 2002 to $8 \%$ in 2004 of drivers using a cell phone on the road at any given time during daylight hours (Glassbrenner, 2005). Cell phones and other in-vehicle devices have the potential to distract drivers, impair their control of the vehicle, and reduce their awareness of the driving environment. Based on an analysis of NHTSA crash data, driver inattention accounted for approximately $25 \%$ of police-reported crashes (Ranney, Mazzae, Garrott, \& Goodman, 2000). The components of inattention-related crashes include distraction, looked but failed to see, and situations where the driver was drowsy or fell asleep. Wiereille and Tijerina (1996) provided additional detail about sources of distraction by searching police report narratives for a set of crashes in the North Carolina accident database. They identified 2,816 crashes in which drivers' attention was diverted. More than half $(55.5 \%)$ of these crashes were related to distraction due to interacting with objects inside the vehicle, another person or animal, or instrumentation.

Structural and cognitive factors contribute to distractions. Structural interference includes holding or dialing a cell phone, and cognitive interference includes actively engaging conversations on a cell phone. Patten, Kircher, Ostlund and Nilsson (2004) compared the effects of hand-held and hands-free cell phones and found that both yielded similar deficits in driving 
performance. This finding suggests that when actively conversing on cell phones, the imposed cognitive load withdraws attention from the processing of information related to safe driving. Strayer, Drews and Johnson (2003) showed that even when participants looked directly at objects in the driving scene while conversing on a hands-free cell phone, they were less likely to "see" them because their attention was directed elsewhere. Drivers appear to be less sensitive to the driving environment when they are cognitively loaded. Understanding the degree to which cognitive load diminishes drivers' awareness of their decreased sensitivity to driving-related information may be a critical factor in mitigating the effect of potentially distracting in-vehicle technology (Lee \& Strayer, 2004).

This decreased sensitivity and the correspondence between actual and perceived sensitivity is the major focus of this study. To explore these issues, a dynamic change blindness paradigm (Zheng, Tai, \& McConkie, 2004) is used to examine the effect of cognitive load on sensitivity. Participants' confidence in detecting changes while driving was compared with their actual detection performance with longitudinal (Experiment 1), lateral (Experiment 2), and parked vehicle changes (in both experiments). We predict that when attention is disrupted by cognitive load the strength of the relationship between sensitivity to changes and confidence in detecting those changes will decrease.

\section{METHOD}

\section{Participants}

Twelve native English speakers, aged 22 to 28 years ( 5 men and 7 women), participated in Experiment 1, and another 12, aged 20 to 26 years ( 3 men and 9 women), participated in Experiment 2. All drivers had normal or corrected-to-normal vision, at least 5 years of driving experience, and a valid driver's license. Participants were paid $\$ 15 /$ hour, with additional compensation (up to \$10) available depending on how well they performed the in-vehicle information task.

\section{Apparatus and Tasks}

Driving simulator. A fixed-based, medium-fidelity driving simulator was used to conduct the experiment. The simulator uses a 1992 Mercury Sable vehicle cab that has been modified to include a 50-degree visual field of view, force feedback steering wheel, and a rich audio environment. The fully textured graphics are generated by DriveSafety's Vection ${ }^{\mathrm{TM}}$ software that delivers a $60-\mathrm{Hz}$ frame rate at 1024 x 768 resolution.

Driving task. Each driving scenario included a straight, four-lane suburban road with a parking lane on each side. Each drive was approximately 6.5 miles long and drivers were asked to maintain a speed of $30 \mathrm{mph}$ and to drive normally.

Change detection task. The change detection task was administered using a dynamic change detection paradigm (Zheng, Tai, \& McConkie, 2004), which included blanking of the projection screen for one second. In half of the drives, participants were informed that the projection screen might blank and a change could occur during the blank. Twenty-five percent blanks were catch trials, in which the screen blanked and no changes occurred. In the other half of the drives, the 
changes occurred without blanking. Each potential change occurred when participants reached pre-designated locations separated by intervals of approximately 200 meters. Participants were asked to indicate the type of change observed by pressing the corresponding button on the steering wheel.

In-vehicle information task. During half of the drives, participants were asked to listen to messages presenting information about the cost, quality, and wait time at three different restaurants (Reyes \& Lee, 2004). The messages were created with a text-to-speech editor using an English male voice engine. After listening to each message twice, participants were asked to verbally answer questions about the message information. A bonus was used to encourage participants to provide their best answers.

\section{Experimental Design and Independent Variables}

Each of the two experiments was a 2 (secondary task) x 2 (blanking) x 3 (change) withinsubjects design. Each participant drove four drives; two included blanking of the screen (blank) and two did not (no-blank). The secondary task was provided in an auditory format and two of the four drives included this secondary task (one with blanking and one without). The order of the experimental conditions was counterbalanced with a Latin square design.

During each drive, there were three types of possible changes to the vehicles in the driving scene. Experiment 1 included longitudinal changes (i.e., vehicles moved forward and backward along the roadway), and Experiment 2 included lateral changes (i.e., vehicles moved between the right lane and left lane). There were also parked vehicle changes (i.e., color, identity, and location on parking lane) in both experiments. Each type of change was encountered 12 times during a drive, and each change occurred during a blank in the blank condition. These 36 changes were presented in a different order in each drive. An additional twelve blanks without any changes occurred in the blank condition to prevent participants from guessing.

\section{Procedure}

At the beginning of each experimental drive, participants were instructed to accelerate to $30 \mathrm{mph}$ and maintain this speed throughout the drive. Participants were required to maintain their position in the center of the right lane. Upon experiencing a change, participants were asked to respond to the type of change as quickly as possible by pressing the corresponding button on the steering wheel. An additional instruction was given to the participants in Experiment 2. A pace car was placed ahead of the subject vehicle in the left lane, and participants were asked to maintain their speed and position relative to the pace car throughout the drives. Participants were also asked to verbally answer the auditory questions as quickly as possible while driving and performing the change detection task. Upon completion of each drive, participants rated their confidence in their ability to detect each type of change. Each experiment took approximately two hours to complete.

\section{Dependent Variables}

Participants' sensitivity to detecting changes was measured by d'. d' was based on the difference between the normalized hit rate and false alarm rate $(\mathrm{z}(\mathrm{H})-\mathrm{z}(\mathrm{F}))$ (Macmillan \& Creelman, 
2005). A hit indicated a change that was correctly identified. A miss was denoted as failing to identify a change correctly. A false alarm (FA) was identified as detection of a change when none was present. A correct rejection (CR) was defined as not responding when there was no change in the blank condition. The change events in the blank condition (for the CR and FA) were matched to equivalent road segments in the no-blank condition so a comparison could be made. Participants' confidence in detecting changes was measured using a subjective rating on a 1 to 10 scale $(1=$ least confidence to $10=$ most confidence $)$.

\section{RESULTS OF EXPERIMENT 1}

A repeated measures ANOVA indicated that participants were less confident in detecting longitudinal vehicle changes when there was a secondary task $(F(1,121)=21.32, p<0.0001)$ and when the screen was blanking $(F(1,121)=8.47, p=0.004)$. The d' data showed somewhat similar patterns. Participants were less sensitive to vehicle changes when there was a secondary task $(F(1,121)=7.63, p=0.006)$ and when the screen was blanking $(F(1,121)=29.97, p<$ $0.0001)$. There was no secondary task $\mathrm{x}$ blanking interaction for either of the dependent variables. The vertical and horizontal lines in Figure 1 show the means for each condition.
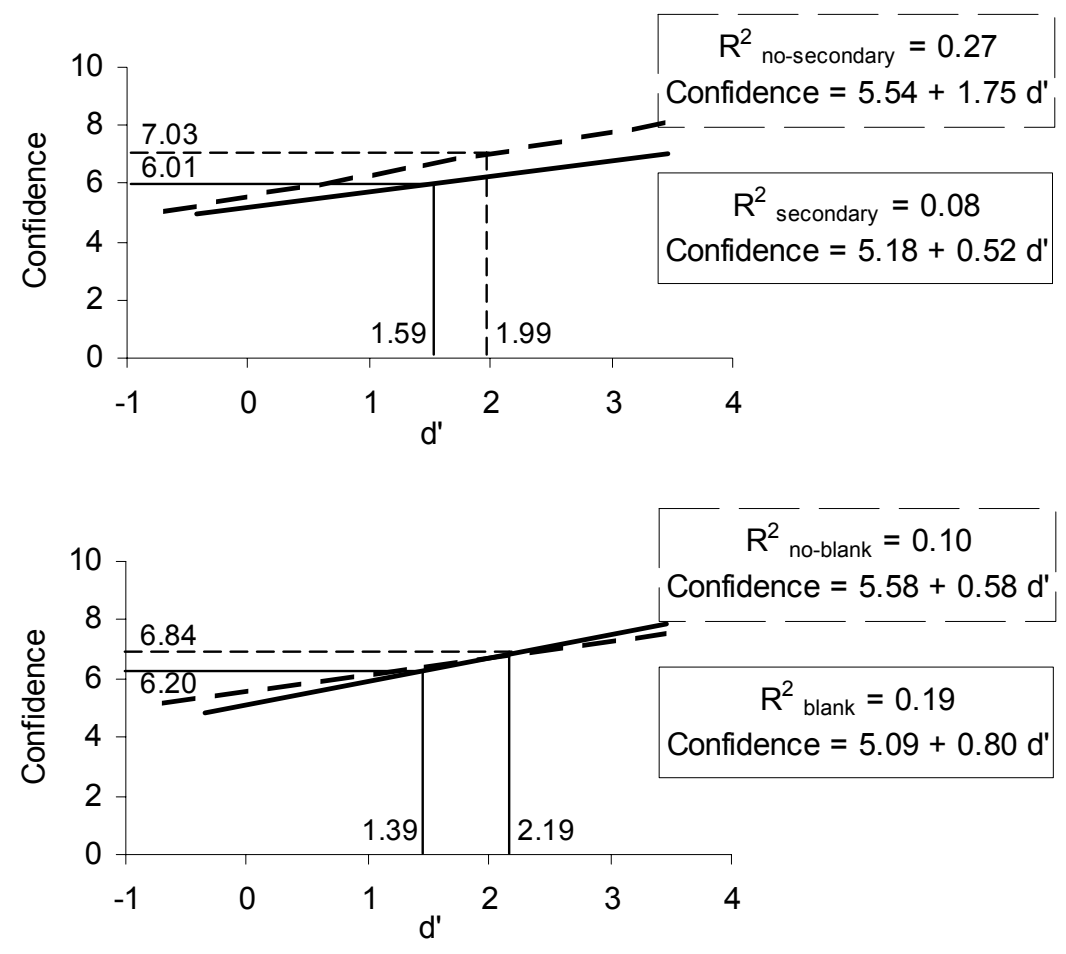

Figure 1. Relationships between d' and confidence for secondary task and blanking conditions in Experiment 1. Dashed lines represent the no-secondary condition in the left panel and the no-blank condition in the right panel.

Correlation and linear regression analyses were used to explore the relationship between d' and rated confidence. Overall, d' and confidence were positively correlated $(r(144)=0.41, p<$ $0.0001)$, suggesting that participants were aware of the factors that influenced their changedetection performance. To further understand the relationship between d' and confidence, regression models were developed for different experimental conditions (Figure 1). Comparison 
of regression coefficients showed that the coefficients were not different between the secondary task conditions $(p=0.479)$ or the blanking conditions $(p=0.892)$. This finding suggests that changes in sensitivity had an overall similar effect on confidence for the effects of secondary task and blanking. However, the correlation coefficient of the no-secondary task condition was marginally higher when compared to the secondary task condition $(p=0.082)$, suggesting that the regression line for the no-secondary task condition fit the data better. The difference for the correlation coefficients of the blank and no-blank conditions did not approach statistical significance $(p=0.386)$.

\section{RESULTS OF EXPERIMENT 2}

Experiment 2 examined the detection of lateral movements of vehicles in a driving scene. A repeated measures ANOVA indicated that participants were less confident in detecting vehicle changes when there was a secondary task $(F(1,121)=31.79, p<0.0001)$ and when the screen was blanking $(F(1,121)=26.87, p<0.0001)$. The interaction between the secondary task and blanking was significant $(F(1,121)=4.33, p=0.039)$, showing that blanking had a greater negative effect on confidence for the secondary task condition than for the no-secondary task condition. For the analysis of d', repeated measures ANOVA indicated that participants were less sensitive to vehicle changes when there was a secondary task $(F(1,121)=16.95, p<0.0001)$ and when the screen was blanking $(F(1,121)=36.58, p<0.0001)$. The interaction between the secondary task and blanking interaction was not significant. The vertical and horizontal lines in Figure 2 show the means for each condition.
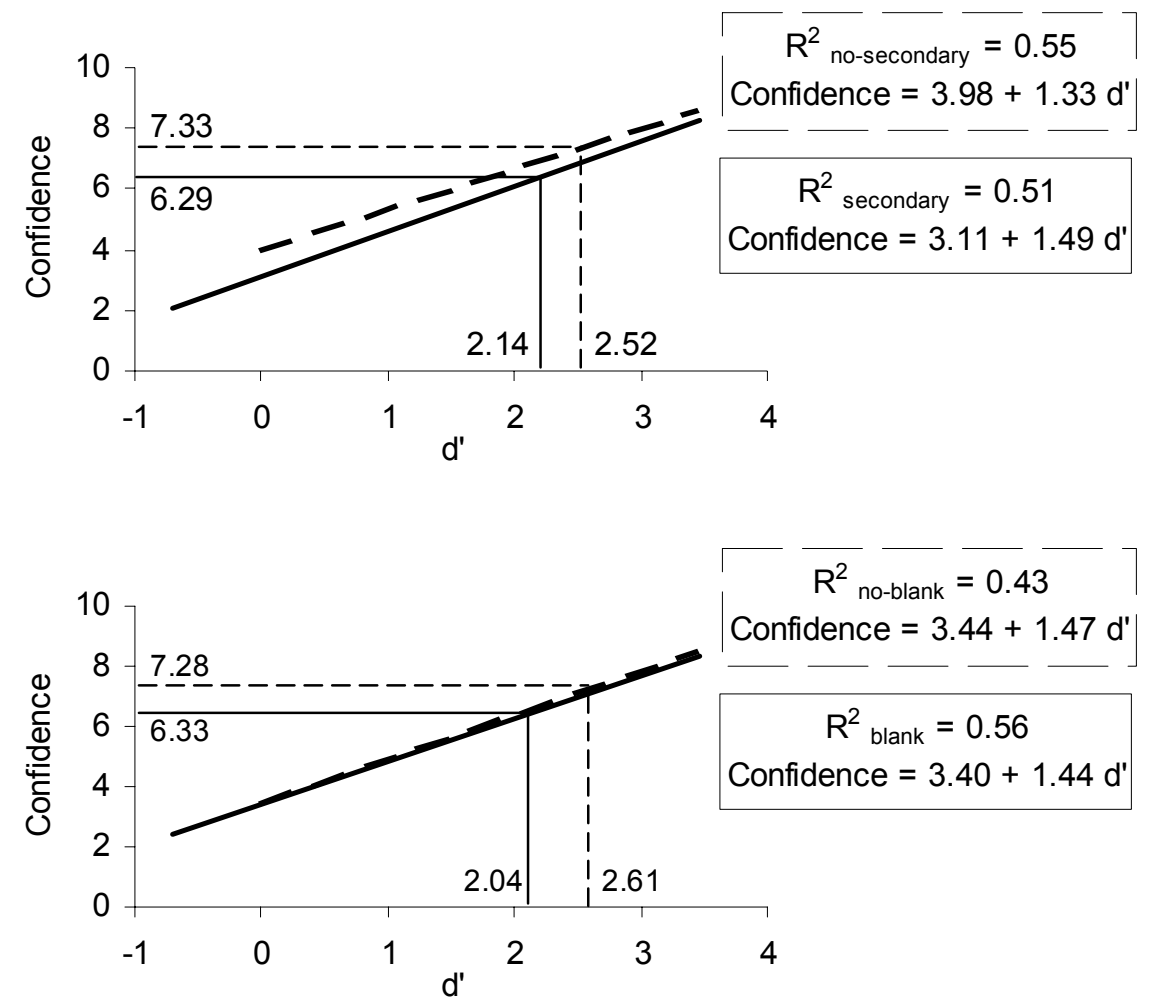

Figure 2. Relationships between d' and confidence for secondary task and blanking conditions in Experiment 2. Dashed lines represent the no-secondary condition in the left panel and the no-blank condition in the right panel. 
Overall, d' and confidence were positively correlated $(r(144)=.73, p<0.0001)$, suggesting that participants were aware of the factors that influenced their change detection performance. Regression models were developed for the experimental conditions (). The regression coefficient comparisons showed that the coefficients were not different between the secondary task conditions $(p=0.479)$ or the blank conditions $(p=0.892)$. Thus, the association between d' and confidence level was not stronger when a secondary task or blank condition was introduced. When testing the differences between the correlation coefficients, we found that they were not different between the no-secondary and secondary conditions $(p=0.749)$ or between the noblank and blank conditions $(p=0.264)$.

Comparing the correlation coefficients for Experiment $1(r=0.41)$ and $2(r=0.73)$ showed a systematic difference $(p<0.0001)$. This finding indicates that the actual and perceived performance was more strongly related for the lateral changes. A follow-up ANOVA suggests that both d' and confidence $(F(1,165)=70.29, p<0.0001, F(1,165)=20.32, p<0.0001$, respectively) were higher for lateral changes compared to longitudinal changes.

\section{DISCUSSION}

Other studies have suggested that observers tended to overestimate their ability to detect changes (Levin, Momen, Drivdahl \& Simons, 2000). Our study found that drivers were aware of how the secondary task and blanking affected their change detection performance. The positive relationship between d' and confidence held true for different experimental conditions.

We hypothesized that the strength of the relationship would decrease as cognitive load increased, and the results somewhat confirmed this hypothesis, as reflected in the marginally significantly lower $\mathrm{R}^{2}$ in the presence of a secondary task in Experiment 1. Similar to Lesch and Hancock's (2004) findings that driver may not be aware of their degraded performance while using a cell phone, our results suggest that drivers' degree of self-awareness is situation-dependent. There is clear evidence that participants were less sensitive and less confident to the longitudinal changes. In this more difficult task, the presence of a secondary task decreased the strength of the association between change detection performance and confidence in detecting changes. The notably low $\mathrm{R}^{2}$ for the secondary condition in Experiment 1 suggests that participants were only vaguely aware of their performance in detecting difficult changes while cognitively loaded. This imprecision may lead drivers to improperly estimate their ability to handle difficult situations when they are cognitively loaded.

These results suggest that providing drivers with feedback regarding the degree of distraction could enhance their ability to manage interactions with potentially distracting in-vehicle devices. This study also suggests that feedback might be most valuable for situations that challenge a driver's attentional capacity.

\section{ACKNOWLEDGMENTS}

The work presented herein is part of the SAfety VEhicle(s) using adaptive Interface Technology (SAVE-IT) program that was sponsored by the National Highway Traffic Safety Administration (NHTSA) (Project Manager: Michael Perel) and administered by the John A. Volpe National Transportation Systems Center (Project Manager: Mary D. Stearns). 


\section{REFERENCES}

Glassbrenner, D. (2005). Driver cell phone use in 2004 - Overall results. Retrieved April 5, 2005, from http://www-nrd.nhtsa.dot.gov/pdf/nrd-30/NCSA/RNotes/2005/809847.pdf

Lee, J. D., \& Strayer, D. L. (2004). Preface to the special section on driver distraction. Human Factors, 46(4), 583-586.

Lesch, M. F., \& Hancock, P. A. (2004). Driving performance during concurrent cell-phone use: are drivers aware of their performance decrements? Accident Analysis and Prevention, 36, 471-480.

Levin, D. T., Momen, N., Drivdahl, S. B., \& Simons, D. J. (2000). Change blindness blindness: the metacognitive error of overestimating change-detection ability. Visual Cognition, 7, 397412.

Macmillan, N. A., \& Creelman, C. D. (2005). Detection Theory: A user's guide (2nd ed.). Mahwah, NJ: Lawrence Erlbaum Associates, Inc.

Patten, C. J. D., Kircher, A., Ostlund, J., \& Nilsson, L. (2004). Using mobile telephones: cognitive workload and attention resourse allocation. Accident Analysis and Prevention, 36, 341-350.

Ranney, T. A., Mazzae, E., Garrott, R., \& Goodman, M. J. (2000). NHTSA driver distraction research: Past, present, and future. Retrieved April 5, 2005, from http://wwwnrd.nhtsa.dot.gov/departments/nrd-13/driver-distraction/PDF/233.pdf

Reyes, M. L., \& Lee, J. D. (2004). The influence of IVIS distractions on tactical and control levels of driving performance. Proceedings of the Human Factors and Ergonomics Society, $48,2369-2373$.

Strayer, D. L., Drews, F. A., \& Johnston, W. A. (2003). Cell phone-induced failures of visual attention during simulated driving. Journal of Experimental Psychology-Applied, 9(1), 23-32.

Wierwille, W. W., \& Tijerina, L. (1996). An analysis of driving accident narratives as a means of determining problems caused by in-vehicle visual allocation and visual workload. In A. G. Gale, I. D. Brown, C. M. Haslegrave \& S. P. Taylor (Eds.), Vision in Vehicles - V: Elsevier Science.

Zheng, X. S., Tai, Y., \& McConkie, G. W. (2004). Exploring drivers' situation awareness in a dynamic traffic environment. Proceedings of the Human Factors and Ergonomics Society, 48, 2374-2377. 